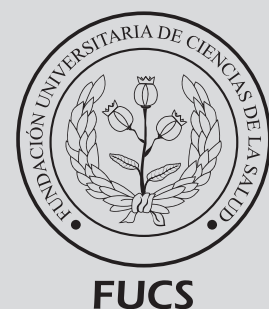

\title{
Estudio de la vacunación contra el COVID-19 a nivel de América
}

\section{Study on COVID-19 vaccination in the Americas}

a Ingeniero. Magister en Gestión de la Tecnología Educativa, Especialista en Administración de la Informática Educativa.

Docente de matemáticas e Investigador, Secretaría de Educación de Soacha, Cundinamarca.

\section{R E S U M EN}

Introducción: una de las expectativas que tenemos actualmente es colocar vacunas seguras y efectivas para suministrar a la población. Con estas vacunas se intenta conseguir la inmunidad colectiva que permita quebrar la cadena de transmisión. Hay que tener estrategias de vacunación frente al COVID-19 teniendo en cuenta la disponibilidad progresiva de dosis, es necesario realizar un ejercicio de priorización atendiendo a principios éticos y criterios de riesgo. Objetivo: comparar si hay diferencias en los promedios de vacunación por cada 100 personas para COVID-19 entre los diferentes países de América. Materiales y métodos: se utilizó la base de datos de la vacunación con la información acumulada al 7 de mayo 2021. Se realizó a partir del análisis estadístico de los datos aplicando el análisis de varianza, se utilizó el paquete estadístico SPSS v.25. Resultados: a partir del análisis estadístico de los datos aplicando el análisis de varianza el p-valor de la prueba para la variable de vacunación es de 0,000, en este caso es menor a $\alpha=0.05$, de esta manera se rechaza Ho, es decir la prueba de análisis de varianza nos indica que hay diferencias significativas entre la vacunación contra COVID-19 para los diferentes países de América. Conclusiones: el panorama ante la vacunación contra el COVID-19 a nivel de América es bastante preocupante, pues hay varios países con un bajísimo nivel de vacunación, esto se puede deber a que no sea una prioridad para todos los países, especialmente para los que tienen una baja densidad de población y no se estén presentando un gran número de contagios y muertes.

Palabras clave: SARS-CoV-2; COVID-19; vacunación; pandemia.

(C) 2021 Fundación Universitaria de Ciencias de la Salud - FUCS. Este es un artículo Open Access bajo la licencia CC BY-NC-ND (http://creativecommons.org/licenses/by-nc-nd/4.0/).

INFORMACIÓN DEL ARTÍCULO

Historia del artículo:

Fecha recibido: mayo 10 de 2021 Fecha aceptado: junio 8 de 2021

\footnotetext{
Autor para correspondencia.

Jorge Enrique Díaz Pinzón jediazp@unal.edu.co
}

DOI

10.31260/RepertMedCir.01217372.1213 


\section{A BSTRACT}

Introduction: one of our current expectations is to supply safe and effective vaccines to be administered to the population. The endpoint is achieving herd immunity through vaccination in order to break the chain of transmission. COVID 19 vaccination strategies expanding access to vaccine doses are necessary. Prioritization on the basis of ethical principles and risk criteria is required. Objective: to compare whether there are differences in COVID 19 vaccination rates per 100 people among the different countries of the Americas. Materials and methods: the vaccination database with the information collected to May 72021 was used. An analysis of variance test was applied using the SPSS v.25 statistical package. Results: the p-value obtained in the statistical analysis of variance test for the vaccination variable was 0.000 , which is less than $\alpha$ $=0.05$, therefore, the hypothesis $(\mathrm{Ho})$ is rejected, which means, the analysis of variance test indicates there are significant differences in COVID-19 vaccination in the different countries of America. Conclusions: the outlook for COVID-19 vaccination in the Americas is quite worrying, as there are several countries with very low vaccination rates, maybe due to the fact vaccination is not a priority for all countries, especially for those with low population density, who are not experiencing high COVID 19 infection and death rates.

Key words: SARS-CoV-2; COVID-19; vaccination; pandemic

(C) 2021 Fundación Universitaria de Ciencias de la Salud - FUCS

This is an open access article under the CC BY-NC-ND license (http://creativecommons.org/licenses/by-nc-nd/4.0/).

\section{INT RO DUCCIÓN}

En diciembre de 2019 en la ciudad de Wuhan, provincia de Hubei en China, se originó un brote de casos de neumonía de origen extraño, enfermedad denominada, al presente como COVID-19. ${ }^{1}$

Una de las expectativas que existen es poder colocar vacunas seguras y efectivas para suministrar a la población. Con ello se intenta conseguir la inmunidad colectiva que permita quebrar la cadena de transmisión. ${ }^{2}$ Hay que tener estrategias de vacunación frente al COVID-19, teniendo en cuenta la disponibilidad progresiva de las dosis, es necesario realizar un ejercicio de priorización atendiendo principios éticos y criterios de riesgo.,4 Estas vacunas se han producido en un tiempo récord comparadas al resto por varios motivos: a) no se partía de cero, ya que se han utilizado tecnologías conocidas y utilizadas antes; b) se han podido cubrir diferentes fases que como norma son secuenciales; c) la etapa de alistamiento que en muchas vacunas dura mucho tiempo, aquí se ha disminuido por la facilidad de inclusión de voluntarios y d) porque debido a la emergencia mundial ha existido una importante colaboración de fondos públicos y privados. ${ }^{5} \mathrm{El}$ continente americano se ha transformado en la región del mundo más perjudicada por la pandemia de COVID-19. La mayor parte de los casos y muertes se ubican en Estados Unidos y Brasil, no obstante, la situación se ha agravado en diversos países de la región en los últimos meses con un impacto catastrófico. Para febrero 2021, se estimaba que 610.000 personas habían fallecido por COVID-19 en Latinoamérica y el Caribe, lo cual representa el $27 \%$ de las fatalidades en todo el mundo. Recientemente Perú, Panamá, México, Colombia y Argentina tienen las tasas de mortalidad más altas. ${ }^{6}$ Diferentes factores han influido en la carga especialmente pesada de la enfermedad COVID-19 en la región de Latinoamérica y el Caribe (LAC).
Entre ellos, se ubican los sistemas de salud amortiguados, así como la pobreza y la informalidad laboral que han hecho indefectibles los confinamientos estrictos. Asimismo, deben considerarse las comorbilidades, en especial la obesidad, diabetes e hipertensión, que tienen una alta prevalencia en la región y se han reconocido como factores de riesgo para complicaciones y muerte por COVID-19. ${ }^{7}$ Permanece claro que la situación discrepa mucho de resolverse en el corto plazo y las medidas de control han sido exiguas. Es en este contexto que las vacunas para COVID-19 aportan una luz de esperanza con el fin de enfrentar esta grave crisis de salud. ${ }^{8}$ Es innegable que después de conseguir el desarrollo de vacunas seguras y eficaces, el siguiente objetivo es lograr su acceso equitativo para todas las personas en el mundo. Sin embargo afronta varios retos entre ellos la producción y suministro global; fragilidades de los sistemas nacionales de compra, planeación y distribución, así como la suspicacia sobre las vacunas alimentada por la epidemia de desinformación. ${ }^{9}$ Hasta ahora se han administrado más de 1.236.122.090 millones de dosis en cerca de 100 países. Es el mayor programa de vacunación de la historia. Las primeras dosis se aplicaron hace poco menos de un año después de que se manifestaran los primeros casos en Wuhan, China. ${ }^{10} \mathrm{El}$ objetivo de esta investigación es comparar si hay diferencias en los promedios de vacunación para COVID-19 entre los diferentes países de América.

\section{METODOLOGÍA}

El trabajo de investigación se verificó mediante un enfoque cuantitativo según Hernández, citado por Díaz ${ }^{11}$, en un proceso secuencial y probatorio; se calculan las variables 
en un determinado contexto; se exploran las mediciones alcanzadas (con frecuencia utilizando métodos estadísticos) y se instituye una serie de conclusiones con respecto a las hipótesis. Todos los experimentos cuantitativos operan un formato estándar con algunas pequeñas diferencias interdisciplinarias para crear una suposición que será probada o desmentida. Esta debe ser justificable por medios matemáticos y estadísticos, para establecer la base en torno de la cual se diseña todo el experimento. ${ }^{12,13}$

\section{POBLACIÓN}

Esta investigación se centró en 40 países de América que han recibido vacunación contra COVID-19, con la información de la $\mathrm{BBC}^{10}$, con corte al 7 de mayo 2021 .

\section{RESULTAD OS}

En la figura 1 se aprecia la dosis por cada 100 personas en América al 7de mayo 2021. En ella se puede apreciar que los países con mayor número de dosis aplicadas contra COVID-19 son: Islas Malvinas (126,5), Islas Caimán $(105,9)$, Bermudas (89,8), Chile (80), Estados Unidos $(75,3)$, Islas Turcas $(64,7)$, Uruguay $(56,8)$, Anguila $(46)$, Canadá $(39,5)$, Dominica $(38,6)$ y Monserrat $(38,2)$. Los países de América con menor número de dosis aplicadas contra COVID-19 son: Honduras $(0,6)$, Venezuela $(0,9)$, Guatemala $(1,0)$, Paraguay $(2,0)$, Nicaragua $(2,0)$, Trinidad y Tobago $(4,2)$, Jamaica $(4,6)$, Perú $(5,7)$, Ecuador $(6,5)$ y Bahamas $(6,5)$.

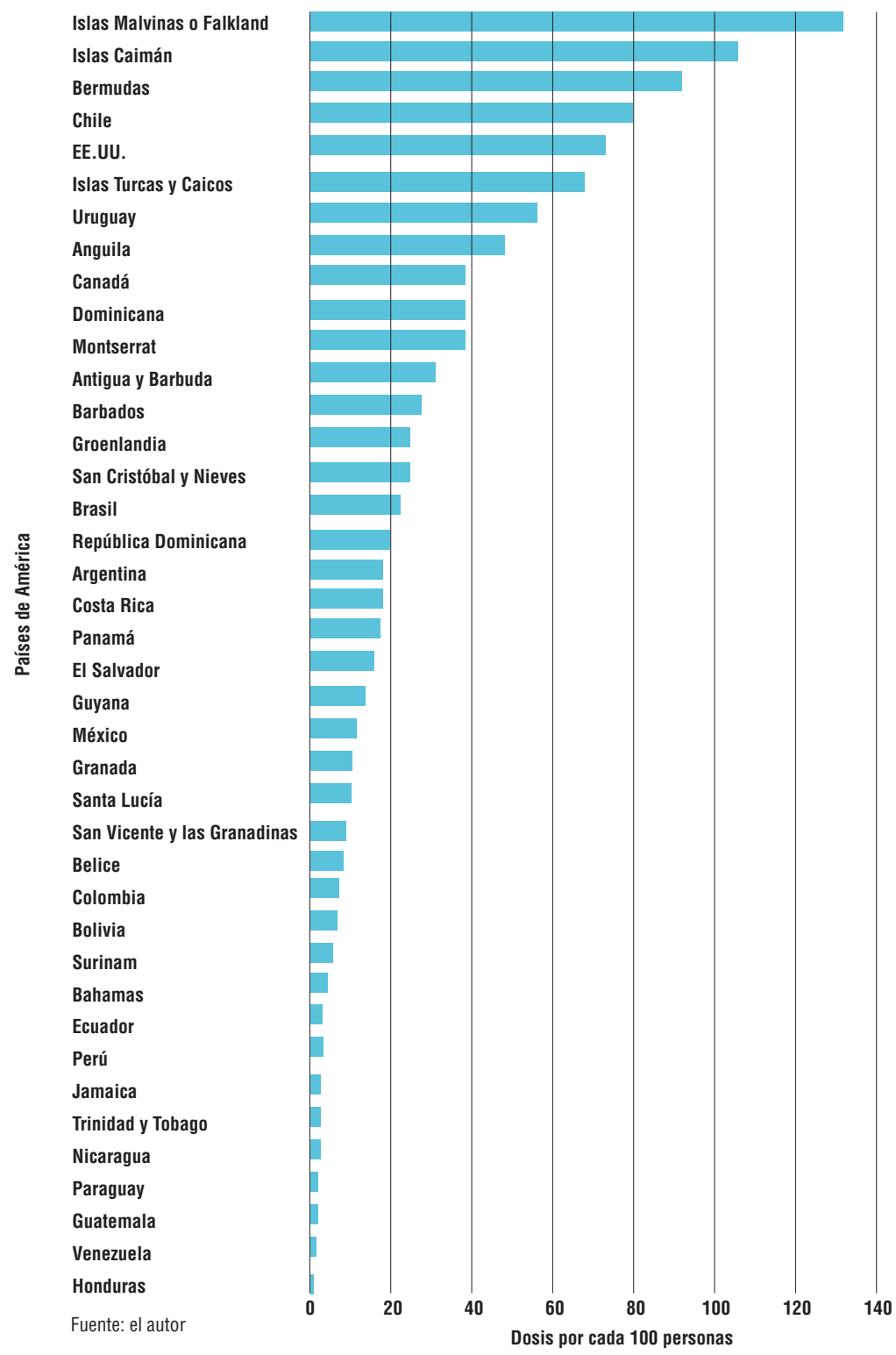

Figura 1. Dosis por cada 100 habitantes en América. Fuente: $\mathrm{BBC}^{10}$ 


\section{ANÁLISIS ESTADÍSTICO}

Se plantearon hipótesis para la prueba de comparación de medias utilizando el análisis de varianza (ANOVA) para la variable vacunación, diseñándose como una relación causal, como se expone a continuación.

Hipótesis alternas (Ha): hay diferencias en el promedio de los puntajes obtenidos por la vacunación contra COVID-19 entre los diferentes países de América.

Hipótesis nula (Ho): no hay diferencias en el promedio de los puntajes obtenidos por la vacunación contra COVID-19 entre los diferentes países de América.
Prueba estadística: el valor de significancia de la prueba es de $\alpha=0.05(5 \%)$, si es mayor se acepta la hipótesis nula, cuando es menor se rechaza la hipótesis nula, se utilizó el paquete estadístico SPSS v.25.

En la tabla 1 se aprecian los diferentes p-valor de la prueba, para la variable vacunación es de 0,000, en este caso es menor a $\alpha=0.05$, de esta manera se rechaza Ho, es decir la prueba de análisis de varianza nos indica que hay diferencias significativas entre la vacunación contra COVID-19 para los diferentes países de América.

Tabla 1. ANOVA-Vacunación-País

\begin{tabular}{|l|ll|l|l|l|}
\hline \multicolumn{7}{|c}{ Suma de cuadrados } & \multicolumn{1}{l}{ gl } & \multicolumn{2}{l|}{ Sig. } \\
\hline Entre grupos & 109226,486 & 39 & 2800,679 & 2801,377 &, 000 \\
\hline Dentro de grupos & 79,980 & 80 & 1,000 & & \\
\hline Total & 109306,466 & 119 & & & \\
\hline
\end{tabular}

Fuente: el autor

\section{CONCLUSIONES}

Se estableció que los países de América con mayor número de dosis aplicadas por cada 100 habitantes contra COVID-19 son: Islas Malvinas (126,5), Islas Caimán $(105,9)$, Bermudas (89,8), Chile (80), Estados Unidos (75,3), Islas Turcas $(64,7)$, Uruguay $(56,8)$, Anguila $(46)$, Canadá $(39,5)$, Dominica $(38,6)$ y Monserrat $(38,2)$. Los países de América con menor número de dosis aplicadas contra COVID-19 son: Honduras $(0,6)$, Venezuela $(0,9)$, Guatemala $(1,0)$, Paraguay $(2,0)$, Nicaragua $(2,0)$, Trinidad y Tobago $(4,2)$, Jamaica $(4,6)$, Perú $(5,7)$, Ecuador $(6,5)$ y Bahamas $(6,5)$. Ahora, a partir del análisis estadístico de los datos aplicando el análisis de varianza, el p-valor de la prueba, para la variable vacunación es de 0,000, en este caso es menor a $\alpha=0.05$, de esta manera se rechaza Ho, es decir la prueba de análisis de varianza nos indica que hay diferencias significativas entre la vacunación contra COVID-19 para los diferentes países de América, fecha de corte 7 de mayo 2021.

El panorama ante la vacunación contra el COVID-19 a nivel de América es bastante preocupante, hay varios países con un bajísimo nivel de vacunación, esto se puede deber a que no sea una prioridad, en especial para los que tienen una baja densidad de población y no presenten en gran número de contagios y muertes. La problemática con este escenario es que mientras el virus progrese en algún lugar, podrá mutar y migrar. Las variantes resistentes a las vacunas proseguirán su evolución. El desarrollo de vacunas para la pandemia de COVID-19 es el resultado de un reciente esfuerzo mundial y simboliza una esperanza para los países de LAC severamente perjudicados por esta enfermedad.
Al presente, es primordial fortificar la capacidad de las autoridades nacionales para establecer las campañas de vacunación, continuar la farmacovigilancia y comunicar a la población la seguridad y efectividad de las vacunas., ${ }^{9,14}$

\section{REFEREN CIAS}

1. World Health Organization. Novel Coronavirus (2019-nCoV): situation report. World Health Organization; 2020. p. 7.

2. Casas I, Mena G. La vacunación de la COVID-19. Medicina Clínica. 2021;156(10):500-2. doi: 10.1016/j.medcli.2021.03.001

3. European Centre for Disease Prevention and Control. COVID-19 vaccination and prioritization strategies in the EU/EEA [Internet]. [Consultado 7 May 2021]; Disponible en: https://www.ecdc. europa.eu/en/publications-data/covid-19-vaccination-andprioritisation-strategies-eueea

4. Ministerio de Sanidad, Consumo y Bienestar Social. Estrategia de vacunación COVID-19 en España [Internet]. [consultado 7 May 2021]; Disponible en: https://www.mscbs.gob.es/profesionales/ saludPublica/ccayes/alertasActual/nCov/vacunaCovid19.htm

5. Jeyanathan M, Afkhami S, Smaill F, Miller MS, Lichty BD, Xing Z. Immunological considerations for COVID-19 vaccine strategies. Nat Rev Immunol. 2020;20(10):615-32. doi: 10.1038/s41577-020-00434-6

6. Statista. Mortality rate due to coronavirus disease (COVID-19) in selected countries in Latin America and the Caribbean as of March 8 [Internet]. 2021 [consultado 7 May 2021]; Disponible en: https:// www.statista.com/statistics/1114603/latin-america-coronavirusmortality-rate/

7. COVID-19 in Latin America: a humanitarian crisis. Lancet. 2020;396(10261):1463. doi: 10.1016/S0140-6736(20)32328-X 
8. Dreser A. Retos y avances en la vacunación contra COVID-19 en Latinoamérica y el Caribe. Salud UIS. 2021;53: e21002. doi: 10.18273/saluduis.53.e:21002

9. Kim J, Marks F, Clemens JD. Looking beyond COVID-19 vaccine phase 3 trials. Nat Med. 2021;27:205-211. doi: 10.1038/s41591021-01230-y

10. Dale B, Stylianou N. Vacunas contra el Coronavirus: ¿Cuál es la situación de tu país? [Internet]. BBC News Mundo; 2021 [consultado 7 May 2021]; Disponible en: https://www.bbc.com/ mundo/noticias-56025727

11. Díaz-Pinzón JE. Estudio de los resultados del contagio por COVID-19 a nivel mundial. Repert Med Cir. 2020;29(Núm. Supl.1):65-71. doi: $\quad$ 10.31260/RepertMedCir.01217372.1089
12. Díaz-Pinzón, J.E. Proyección del COVID-19 en Colombia. Rev. Med. 2020;28(1):11-20. doi: https://doi.org/10.18359/rmed.4702

13. Díaz-Pinzón, J.E. Soporte técnico de simulación Phet en la enseñanza y aprendizaje de fracciones equivalentes. Revista de investigaciones Universidad del Quindío. 2016;28(2):31-41. doi: https://doi.org/10.33975/riuq.vol28n2.6

14. Laxminarayan R, Fitzpatrick S, Levin S. How to Build Trust in Covid-19 Vaccines. Why people distrust vaccines and how they can be convinced otherwise. Nautilus [Internet]. Nautilus 2020 [consultado 7 May 2021]; Disponible en: https://nautil.us/ issue/93/forerunners/how-to-build-trust-in-covid_19-accinesen: https://es.slideshare.net/lualberts20/medidas-de-frecuencia-enepidemiologa-2015 